\title{
Investigating the Role of Medical Terms Translation in Facilitating Learning Process for Medical Students
}

\author{
Abdelrahman Elyass Mohamed Abdelmajd \\ University of Khartoum-Faculty of Arts, Sudan \\ e-mail:elyass572003@yahoo.com \\ Hassan Mahill Abdallah Hassan \\ Sudan University of Science \&Technology, Sudan \\ e-mail:mahill74@yahoo.com
}

\begin{abstract}
This paper aims to answer "To what extent Medical Terms Translation can help in Facilitating Learning Process for Medical Students?" The researcher has adopted the quantitative method as well as the questionnaire as a tool for collecting data relevant to the study to bring to the light the importance of Medical Terms Translation in Facilitating Learning Process for students who study Medical Sciences. The sample of this study comprises (60) students. The marks obtained from the questionnaire were compared. From one hand, the results have revealed that the students encountered difficulties in understanding the Medical Terms. The result has shown that the questionnaire significance indicates that there is highly difference among students. Therefore, this indicates that Medical Terms Translation can play great role in Facilitating Learning Process for Students who study Medical Sciences.

Keywords: EMI , CEMIT, EMT, MTQ, QMTI, VRMT
\end{abstract}




\section{INTRODUCTION}

Although translation does not occupy the most central position in the world of medicine, it certainly plays an important role in knowledge mediation: sharing medical research results, publicizing new findings in the international scientific community and marketing new medicinal products and medical devices are key features of this role (cf. Andriesen 2006; Montalt-Resurrecció and Shuttle worth 2012). Apart from books, articles and presentations, translated medical texts primarily include registration documents, such as application dossiers for the registration of new medicines and medical devices, as well as instruction manuals for medical equipment and instruments, and documents for clinical trials. Medical translators who are responsible for mediating professional communication are expected to have considerable expertise in translation and in a given subject area. What is more, written materials, such as health surveys, patient consents, posters, leaflets etc. need to be made available to foreign patients whose command of a given language may be insufficient. This is frequently performed by medical translators who specialise in professional-layman communication. A separate group of facilitators is constituted by public service interpreters, who mediate communication between professionals, and also between healthcare professionals, e.g. hospital staff and patients. Therefore, medical translators and interpreters facilitate the communication process between patients and medical professionals because the failure to communicate with a patient may lead to health or life-threatening situations if a physician is unable to obtain information from a patient (cf. Heine 2003). Medical translation plays an ancillary role in medical research and practice, but it is also worth remembering that medicine is one of the major areas which involve translation:

Medical translation may well be the most universal and oldest form of scientific translation because of ubiquitousness of human anatomy and physiology (after all, the human body is much the same everywhere), the long, venerable and well-documented history of medicine, and the hitherto uniform character of the language of medicine, at least in the West (Fischbach 1998:1). There is a growing tendency in healthcare communication to focus attention on the patient (Montalt 2012:13; Krystallidou 2012:74-95) and it is now generally acknowledged that there is a need for qualified medical translators and interpreters to facilitate mediation in interlingual and intercultural medical settings, resulting in a number of translators specialising in this particular area worldwide. It appears, however, that the quality of medical translation is subject to improvement, which particularly concerns translator training and qualifications, as well as verification measures applied to detect translation errors in medical texts. That is because quality becomes an issue of vital significance when a translation or interpreting error affects the quality of medical care or reliability of data gathered in the course of clinical trials.

The purpose of this paper is to discuss the importance of Medical Terms Translation in Facilitating Learning Process for Medical Students, to present various methods of quality assurance in medical translation, to reflect on the qualifications of medical translators and to provide suggestions regarding medical translation quality assurance. 


\subsection{Aims and Scope of the Study}

This study aims to investigate the Role of Medical Terms Translation in Facilitating Learning Process for Medical Students. The scope of the study is limited to second class students of Medical Science at Khartoum College for Medical Science. There are (60) second class students of Medical Science at Khartoum College for Medical Science.

\section{LITERATURE REVIEW}

\subsection{Consequences of Errors in Medical Interpretation and Translation (CEMIT)}

The reason why the quality of medical translation and interpreting is so emphasized is the fact that a translation error may trigger severe clinical consequences (cf. Márquez Arroyo 2007:74). Translation errors in scientific articles and presentations may affect an author's reputation. Healthcare services may be adversely influenced, if translation (or interpretation) is misleading either for a physician or a patient, or if vital piece of medical information fails to be translated accurately and, as a result, a patient's condition may not be diagnosed or treated properly.

\subsection{Errors in Medical Interpretation (EMI)}

The main focus of this paper is on medical terms translation, it is also worth noting the significance of accuracy in interpreter-mediated healthcare communication. Flores et al. (2003) studied interpreting errors, their frequency, categories and potential clinical consequences. Their analysis of audio recordings and transcripts of pediatric encounters in a hospital outpatient clinic shows that errors in medical interpretation are quite common. The average number of errors was 31 per session, and $63 \%$ of all errors had potential clinical consequences. Errors were divided into the following five categories - omission, addition, substitution, editorialisation, and false fluency:

1. Omission: The interpreter did not interpret a word/phrase uttered by the clinician, parent, or child.

2. Addition: The interpreter added a word/phrase to the interpretation that was not uttered by the clinician, parent, or child.

3. Substitution: The interpreter substituted a word/phrase for a different word/phrase uttered by the clinician, parent, or child.

4. Editorialisation: The interpreter provided his or her own personal views as the interpretation of a word/phrase uttered by the clinician, parent, or child.

5. False Fluency: The interpreter used an incorrect word/phrase, or word/phrase that does not exist in that particular language (Flores et al. 2003: 7).

The analysis reveals that the largest number of mistakes are made by ad hoc interpreters - random mediators who are not trained in translation or medicine, e.g. children, other family members or bilingual hospital staff. What is more, in comparison to hospital interpreters, ad hoc interpreters made more mistakes which could potentially have clinical consequences, and the most frequent type of error 
was omission. This included omitting questions about allergies to medical products, omitting dosage instructions, frequency or duration of drug administration, omitting significant information about a patient, such as facts from medical history, symptoms or other key information concerning the patient's condition, confusing drug administration routes, advising a patient not to answer personal questions, etc. (Flores et al. 2003).

The results of the study seem to be an argument for stricter rules of selecting interpreters, who need to have appropriate skills and expertise. What is interesting, interpreting errors tend to result from lack of attention or insufficient command of language, rather than cultural differences (Felberg and Skaaden 2012), which means that they could be reduced by adequate training, peer observation and feedback sessions. Thus, their potentially dangerous clinical consequences could be avoided. The study results also indicate that medical interpreters are expected to have adequate command of both source and target languages, and to provide very precise and neutral interpretation of the message -without omissions, additions or expressing an interpreter's personal views, which are categorised as errors by Flores et al. (2003). This approach means that frequently, total accuracy is expected of medical interpreters, with very little tolerance for any reformulations they might want to use. One of the reasons for the 'total accuracy' demand is the concern that knowledge may be distorted in intercultural communication (Montalt 2012:18). Precise rendition is what protects the translated message from being distorted. Frequently, however, demanding accuracy is simply not sufficient: an interpreter should receive adequate training and support in his or her professional development.

\subsection{Errors in Medical Translation (EMT)}

An incident which exemplifies a health-threatening potential of an error in written medical translation is a series of knee replacement surgeries, described by Fakler et al. (2007). The operations involved an erroneous use of the knee prosthesis in Germany in the years 2006-2007, as a result of which 47 people were harmed. Two different types of that knee prosthesis are available - for use with or without cement. The source-language label on the package of the prosthesis included the information that the femoral component was "non-modular cemented," which was incorrectly translated as "non-cemented" or "without cement" (Fakler et al. 2007: 1). For over 12 months, medical professionals who performed or assisted in the operations were unaware of the fact that prosthesis elements had not been implanted in the correct manner. In this particular case patients suffered as a result of a very basic translation error, but it should be noted that it was the combination of human and system failure which contributed to this series of health-threatening incidents. Apparently, control mechanisms had either failed or had not existed at all (cf. Fakler et al.2007:3). This case is merely an example which illustrates the potential clinical impact of an error in a medical translation undetected due to insufficient translation quality control. It is not only mistranslation that may have clinical consequences. Readability is also a critical issue. Patients do not follow written treatment guidelines when they lack clarity (Nisbeth and Zethsen 2012), which can be the case 
in translated patient information leaflets (PILs). Failure to comply with recommended use instructions may be potentially health-threatening.

Adequate quality assurance procedures thus help eliminate errors and improve clarity of translated medical documents by ensuring the provision of qualified professionals and control measures for the detection of mistranslations, discrepancies or cohesion issues.

\subsection{Medical Translation Quality (MTQ)}

Translation quality is a complex issue because, apart from accuracy and correct language use, it involves such factors as client satisfaction and compliance with contractual requirements, and is largely determined by text type, function and expectations in the community related to translation (Gouadec 2010, EN 15038). Medical translation quality assurance involves designing efficient control methods for error detection, readability testing and commissioning adequately qualified professionals to perform medical translation. Hence, one of the critical issues seems to be what qualifications (and training) are required of professional translators of medical texts, and what steps can be taken in order to ensure adequate translation quality. There are no generally applied regulations concerning medical translation, or translator training; there are however guidelines developed by translators' associations, including the International Medical Interpreters Association (IMIA), developed to support translators, interpreters and their clients.

\subsection{Qualifications of Medical Translators and Interpreters (QMTI)}

Particular qualifications which should be required of medical translators and interpreters are subject to debate, and there are controversies regarding academic (medical or pharmaceutical vs. linguistic) background of medical translators (Fischbach 1998; O’Neil 1998; IMIA 2009; Nisbeth Jensen and Korning Zethsen 2012). It is indisputable, however, that handling medical translation demands specific skills.

According to IMIA, medical documents should be translated by professionals who have "a native or near-native, formal level of language proficiency, analytical capabilities, and deep cultural knowledge in the source and target languages" (2009:3), at least college level formal education in the source and target languages, preferably including translation theory and practice. Such professional medical translators should have expert knowledge of the subject matter terminology, understand the source text (thereafter ST), have proficient writing skills and adequate skills in using specialised, professional dictionaries and glossaries. Their professional expertise should also include the ability to conduct terminology research (IMIA 2009:3).

Translating medical texts, just like translating any other text, requires writing skills, while writing is not usually the key feature of medical curricula, and neither are translation strategies. Even though this is still widely debated, linguistic proficiency seems to be necessary because a target text (thereafter TT) produced by a physician with no theoretical training in medical writing may not be sufficiently reader-friendly (O'Neil 1998:73). 
A medical translator's command of medical English and his or her writing skills should also involve a range of genres and registers. A translator should be able to transfer medical information for patients in a way which will foster understanding, i.e. without using unnecessary jargon, complicated syntax, or rarely used vocabulary. Translating documents which are written for medical professionals, on the other hand, requires specific terminology and discourse markers typical of similar texts produced in the target language. Therefore, a translator's linguistic competence involves general and specialised languages. Ideally, a medical translator would not be a medical professional, but an especially trained translator, i.e. a linguist who underwent appropriate training, a view which is also supported by IMIA (2009:4-5).

One reason why it would be unrealistic to expect every medical text to be translated by a specialist medical professional is that "there will always be more medical translations than can be handled by the relatively few physicians who translate [and] medical translation will perforce be done by non-physicians" (O'Neil 1998:69).What is more, medical texts concern a range of areas of medicine and pharmacology, therefore it would be even more difficult for translation assignments to be completed successfully if only an oncologist could translate a text on cancer, or only a cardiologist could be commissioned to translate documents for cardiac patients, etc.(O'Neil 1998:75). On the other hand, the level of expertise required to understand the ST may also be so high that cooperation with a professional who specialises in this particular area may be necessary. What is beyond any dispute is the fact that a medical translator needs to have some back ground knowledge of medicine to make sure that a message is transferred without distortions, which is one of the critical issues in interlingual and intercultural knowledge mediation (MontaltResurrecció and Shuttle worth 2012).

Formal medical training is only one way of developing medical knowledge necessary for adequate translation. Some information on the background of medically knowledgeable linguists is provided by the results of a survey Marla O'Neil (1998) conducted among translators who are not physicians, but specialise in medical translation. Out of those 33 translators ("medically knowledgeable linguists") 5 studied medicine or participated in medical courses, 7 worked in a position which was indirectly related to healthcare or medicine, 6 participated in translation courses with particular focus on medical translation, 25 had access to medical professionals, 11 were close relatives of a medical professional, 6 had a medical condition which resulted in doing background research and contact with medical professionals (the sum of the figures is higher than 33 because respondents could provide more than one answer).Significantly, most respondents admitted that their work was hardly ever proofread. What is more, even if proofreading was performed, it was not always undertaken by a medical or healthcare professional. The reality of medical translation shows that translators must assume sole responsibility for the quality and accuracy of medical translations, which seems to be one of the factors behind the often poor or substandard quality of medical translation, rather than merely the question of medical versus linguistic educational background of the translator. 
In the absence of medical translation certification scheme, medical translators themselves need to decide if they are qualified enough to perform the specialised translation tasks that they are considering to take on. On the other hand, it seems that both a medical professional and a medically knowledgeable linguist can successfully translate medical texts, provided they have sufficient skills, training and experience. The ideal pattern would involve medical professionals editing texts translated by linguists and linguists editing texts translated by medical professionals, as the quality of medical translation can be assured by means implementing special standards or procedures for error control to support competent translators.

\subsection{Verification and Review in Medical Translation (VRMT)}

To improve medical translation quality, a translation process can be designed. It would involve a pre-translation preparation and analysis of the ST, its actual translation and multi-step verification of the TT. The verification process should not only involve error detection, but also conventions and requirements regarding various text types and functions (cf. Mobaraki and Aminzadeh 2012), including readability and clarity in expert-lay communication.

IMIA (2009:6-11) suggest the following steps in the translation process: first the final (electronic) version of the ST is prepared -it is crucial that the ST is 'final,' as this will reduce the risk of errors or ambiguity in the TT. Poorly written or confusing passages are likely to be as poor or confusing in the target language. IMIA also suggest that medical STs should be devoid of figurative language and ambiguity. They also should be culture-neutral, except for documents addressed to specific audiences, e.g. segregated by gender or age group, or those intended for specific, for instance educational or marketing, purposes. Therefore, a pretranslation process should ideally be designed to verify that a final version of a source text is sent to the translator.

In the next step of the translation process a translator reads the text and decides if she or he is qualified to translate the text, i.e. render its meaning accurately, which is followed by the essential stage of translation (IMIA 2009: 8). The translation should be meaning for meaning rather than word for word, it should be precise, accurate, natural and correct in terms of language use: syntax, grammar, spelling, and terminology.

Finally, the TT is subject to verification: the translated document is reviewed and edited by another professional, who ideally should have more subject area expertise and be more experienced than the translator. It is then proofread, ideally by a third person. Both review and proofing should be done by means of careful comparison of the two language versions (IMIA 2009). It may be necessary to adapt the TT to local, for example legal, requirements concerning an informed consent form (ICF) and other medical documents (Fernández Piera and Ardura Ortega 2012: 291). This, however, is provided by client's own specialists rather than a translator, and it certainly should never be considered without consulting the client.

The model of a translation process with multi-step verification by two professionals ensures sufficient measures for quality assurance; however, it is not economical and can be time-consuming. This may mean that yet another condition 
for improving the quality of medical translation is client education. Another crucial aspect of quality assurance is negotiating realistic deadlines and budgets. Obviously, if a text is to be translated and then verified by two professionals, the budget and deadline should be agreed with consideration to realistic time and money necessary to perform all tasks involved in the translation and editing process. The abovementioned multi-step verification model for medical translation is one of several solutions developed for quality assurance. A popular method of translation review is back-translation, i.e. translating the TT 'back' into the source language. It is important that back-translations should be provided by an independent translator who did not handle the original 'forward' translation of a given text. IMIA advise against applying back-translation as a method for verification for the reason that it might not reveal "the target language contextual and usage nuances" (IMIA 2009:2) and, if the text is translated literally by the first translator, its back-translation may seem appropriate. What may also appear as an inaccurate rendition in the backtranslation is frequently an adaptation made by the translator which fully conveys the meaning of the ST, but is lost in back-translation (IMIA 2009:3).

However, the blind back-translation technique is frequently used to verify the accuracy of translation (cf. Andriesen 2006, Fernández Piera and Ardura Ortega 2012), and its advantages have been subject to research (Berkanovic 1980; Andriesen 2006). Berkanovic (1980) conducted a study on the effect of language of a health survey on patients' responses. It involved an analysis of Spanish speakers' responses to the Spanish version of a survey which was a translation of an English ST in comparison with the responses given by another group of Spanish-speaking patients during the same interview, but in English. The results show that there are considerable differences between the two groups in the perceived seriousness of patient's medical condition and the perceived efficacy of care. In the group which was asked questions from the Spanish language version of the survey, those parameters were substantially lower than in the English version interviews. The back-translation revealed that the Spanish language version sounded unnatural: according to the opinion of the person who performed the back-translation, the general meaning of the survey would be understood, but they would be perceived as clumsy, if not funny. Therefore, it could be concluded that the respondents may have taken the English language interview more seriously than the Spanish language interview. Back-translations would have allowed for the identification and correction of unnatural language (Berkanovic 1980). On the other hand, it can be claimed that the double verification process suggested by the IMIA would also be a useful tool here, even without applying the blind back-translation technique. The back-translation method is widely used in the sector of medical research and clinical trials, as it is required by Ethics Committees and regulatory authorities in a number of countries, but it should not be implied that the sole purpose of back-translation is compliance with formal requirements. If it is handled in a professional manner, it is an effective error detection tool (Andriesen 2006:15-16).

The International Society for Pharmacoeconomics and Outcomes Research (ISPOR) has developed a complex review method consisting of performing two parallel forward translations, which are later compared in order to reconcile both 
versions, then performing two back-translations, which are also compared and reconciled. This is followed by a review and harmonization of the translated text. As effective as the method appears in the role of an error detection measure, it is both costly and time-consuming (Andriesen 2006:15-16).

One step of the verification model applied by ISPOR -i.e. parallel translations - also constitutes a separate review method. Two professionals translate the ST simultaneously and a third person (or one of the translators) compares the two versions and makes all the necessary adjustments. As a result, the final TT is a compilation of the best parts of the two parallel translations. This method also generates additional costs, but: "in this case the additional cost has a much more direct and positive effect on the quality of the final document than is the case with a back-translation" (Andriesen2006:16).

Yet another quality management system is used by FACIT, an organisation which manages the preparation, distribution and interpretation of questionnaires which measure health-related quality of life of patients with chronic illnesses. FACIT adopt a complex, multi-step procedure which involves two independent 'forward' (initial) translations, reconciliation of the translations, back-translation, independent reviews performed by 3-4 experts, formatting, proofreading and pilot testing with patients. The process involves a number of linguists and medical specialists, and it favours quality over deadlines and budget restrictions.

The procedures described above, which help ensure sufficient quality of translation and avoid errors which may cause clinical consequences, are not always implemented. In fact, translators admit that their work is rarely or never verified (cf. O'Neil 1998:72), which means that they assume sole responsibility for the quality of their translation. It is worth noting that IMIA Guidelines (2009)imply that the translators themselves are also responsible for assessing whether their level of competence is sufficient for a particular translation task. There are certain steps translators can take in order to maintain a satisfactory level of medical translation. It should not be forgotten that the first step in translation verification is the "final eye,'i.e. the translator's own editing just before the text is deemed ready and sent to undergo subsequent steps of quality assurance process. It requires the capacity for a critical attitude towards a translator's own work. Taking into consideration medical translation quality problems discussed in this paper and presented in other works (e.g. Berkanovic 1980; Andriesen 2006; Fakler et al. 2007; Márquez 2007) it seems that several recommendations can be made to translators to help them avoid potential errors.

\section{RESEARCH METHODS}

The researcher has used the descriptive analytical and quantitative methods as well as a questionnaire as a tool in the collection of relevant data and information in pursuing this paper. Population of this study is drawn exclusively from Khartoum College for Medical Sciences. The population is second class students of Medical Science at Khartoum College for Medical Sciences. A sample of (60) students was randomly selected for the questionnaire. 


\section{FINDINGS AND DISCUSSION}

The researcher has used the questionnaire as a tool in the collection of data relevant to this study. The researcher has designed a questionnaire to answer the question" to extend Medical Terms Translation can help in Facilitating Learning Process for Medical Students? Among the Post second class students who study Medical Sciences to provide answers to the problems that encountered them. The tables below are going to illustrate what has been stated earlier.

\subsection{Teachers' Questionnaire}

This item tries to elicit information from the Medical students concerning their views about role of Medical Terms Translation in Facilitating Learning Process for Medical Students.

Table 1: The use of $\mathrm{L} 1$ equivalences of Medical terms is important in hospital and Medical centres services

\begin{tabular}{|l|l|l|l|l|}
\hline Parameters & Frequency & Percent & Valid Percent & $\begin{array}{l}\text { Cumulative } \\
\text { Percent }\end{array}$ \\
\hline Agree & 54 & $90 \%$ & $90 \%$ & $90 \%$ \\
\hline Neutral & 00 & $00 \%$ & $00 \%$ & $100 \%$ \\
\hline Disagree & 06 & $10 \%$ & $10 \%$ & \\
\hline Total & 60 & $100 \%$ & $100 \%$ & \\
\hline
\end{tabular}

Table (1) above shows that a vast majority of the respondents (90\%) Strongly agree and agree that the use of L1 equivalences of Medical terms is important in hospital and Medical centres services. Only 06\% do not agree to that. This indicates that the use of L1 equivalences of Medical terms is important in hospital and Medical centres services.

Table 2: The use of Arabic Medical equivalences brings in a dependence on L1 among students at the expense of $\mathrm{L2}$

\begin{tabular}{|l|l|l|l|l|}
\hline Parameters & Frequency & Percent & Valid Percent & $\begin{array}{l}\text { Cumulative } \\
\text { Percent }\end{array}$ \\
\hline Agree & 38 & $63.3 \%$ & $63.3 \%$ & $63.3 \%$ \\
\hline Neutral & 00 & $00 \%$ & $00 \%$ & $100 \%$ \\
\hline Disagree & 22 & $36.7 \%$ & $36.7 \%$ & \\
\hline Total & 60 & $100 \%$ & $100 \%$ & \\
\hline
\end{tabular}

Table (2) above shows that a vast majority of the respondents (63.3\%) Strongly agree and agree that the use of Arabic Medical equivalences brings in a dependence on L1 among students at the expense of L2. Only 22\% do not agree to that. This indicates that the use of Arabic Medical equivalences brings in a dependence on L1 among students at the expense of L2. 
Table 3: The use of Latin/English is the best method for teaching Medical Terminologies

\begin{tabular}{|l|l|l|l|l|}
\hline Parameters & Frequency & Percent & Valid Percent & $\begin{array}{l}\text { Cumulative } \\
\text { Percent }\end{array}$ \\
\hline Agree & 30 & $50 \%$ & $50 \%$ & $50 \%$ \\
\hline Neutral & 3 & $05 \%$ & $05 \%$ & $100 \%$ \\
\hline Disagree & 27 & $45 \%$ & $45 \%$ & \\
\hline Total & 60 & $100 \%$ & $100 \%$ & \\
\hline
\end{tabular}

Table (3) above shows that a vast majority of the respondents (50\%) Strongly agree and agree that the use of Latin/English is the best method for teaching Medical Terminologies. Only $45 \%$ do not agree to that. This indicates that the use of Latin/English is the best method for teaching Medical Terminologies.

Table 4: The use of Latin/English/Arabic is the best method for teaching Medical Terminologies

\begin{tabular}{|l|l|l|l|l|}
\hline Parameters & Frequency & Percent & Valid Percent & $\begin{array}{l}\text { Cumulative } \\
\text { Percent }\end{array}$ \\
\hline Agree & 32 & $53.3 \%$ & $53.3 \%$ & $53.3 \%$ \\
\hline Neutral & 12 & $20 \%$ & $20 \%$ & $100 \%$ \\
\hline Disagree & 16 & $26.7 \%$ & $26.7 \%$ & \\
\hline Total & 60 & $100 \%$ & $100 \%$ & \\
\hline
\end{tabular}

Table (4) above shows that a vast majority of the respondents (53.3\%) Strongly agree and agree that the use of Latin/English/Arabic is the best method for teaching Medical Terminologies. Only $26.7 \%$ do not agree to that. This indicates that the use of Latin/English/Arabic is the best method for teaching Medical Terminologies.

Table 5: The use of $\mathrm{L} 1$ minimizes the use of and exposure to $\mathrm{L2}$

\begin{tabular}{|l|l|l|l|l|}
\hline Parameters & Frequency & Percent & Valid Percent & $\begin{array}{l}\text { Cumulative } \\
\text { Percent }\end{array}$ \\
\hline Agree & 43 & $71.7 \%$ & $71.7 \%$ & $71.7 \%$ \\
\hline Neutral & 17 & $28.3 \%$ & $28.3 \%$ & $100 \%$ \\
\hline Disagree & 00 & $00 \%$ & $00 \%$ & \\
\hline Total & 60 & $100 \%$ & $100 \%$ & \\
\hline
\end{tabular}

Table (5) above shows that a vast majority of the respondents $(71.7 \%)$ Strongly agree and agree that The use of L1 minimizes the use of and exposure to L2. Only $00 \%$ do not agree to that. This indicates that the use of L1 minimizes the use of and exposure to L2.

The data collected was analyzed in relation to the study hypothesis. The data was collected by questionnaire which had given to Medical students who do not know answers.

Also analyzing the data collected from the questionnaire which had been given to Medical students exclusively drawn from Khartoum College for Medical 
Sciences. The population is second class students of Medical Science at Khartoum College for Medical Sciences. A sample of (60) students was randomly selected for the questionnaire.

The researcher has used the questionnaire as a tool in the collection of data relevant to this study. The researcher has designed questionnaire to identify the problems encountered Medical students who do not know the answers.

The marks obtained from the questionnaire for all participants were analyzed and compared statistically by using frequencies and percentages. The analysis showed that the highest percentage which is represented agree is estimated by $(69.6 \%)$ in contrast to the percentage of disagree which is estimated by $(19.8 \%)$ lesser than this one. Accordingly, this justifies that there is statistical difference in terms of Medical students' point of views that Medical Terms Translation can play great role in Facilitating Learning Process for Medical Students.

\section{CONCLUSION}

After the comparing and calculation of the sub-hypotheses; we have found that the total number of the five sub-hypotheses percentage $(90 \%+63.3 \%+53.3 \%+71.7 \%)$ equal (69.6\%) which represents negative respond justifies that Medical Terms Translation can play a great role in Facilitating Learning Process for Students who study Medical Sciences.

\section{References}

Andriesen, Simon(2006). "Quality Control in Medical Translations.” Innovations in Pharmaceutical Technology. On line at: http://www.medilingua.com/pdf/QualityControlInMedicalTranslation_IPT200 8.pdf (consulted 23.04.2013).

Berkanovic, Emil (1980). "The effect of inadequate language translation on Hispanics' responses to health surveys." American Journal of Public Health, $70,1273-1281$.

EN 15038 (2006). Translation services — Service requirements. Brussels: European Committee for Standardization.

FACIT(2003) Translation Project Procedures and Guidelines. On line at:http://facitorg000.web108.discountasp.net/translation/trans_guidelines.pdf (consulted 17.04.2013).

Fakler, Johannes $\mathrm{K}$ et al.(2007). "Errors in handling and manufacturing of orthopaedic implants: the tip of the iceberg of an unrecognizedsystem problem?" Patient Safety in Surgery 1 (1), 5.

Felberg, Tatjana and Skaaden, Hanne(2012). "The (de)construction of culture in interpreter-mediated medical discourse." Vincent Montalt-Resurrecció and Mark Shuttleworth (eds.) (2012). Translation andknowledge mediation in medical and health settings. Linguistica Antverpiensia New Series -Themes in Translation Studies11/2012, 95-112.

Fernández Piera, María and Ardura Ortega, Mónica(2012). “Adaptación del consentimiento informado a la legislación española en los ensayos clínicos internacionales." Panace36 (13), 291-293. 
Fischbach, Henry(ed.) (1998). Translation and Medicine.Amsterdam/Philadelphia: Benjamins.

Flores, Glenn et al. (2003). "Errors in medical interpretation and their potential clinical consequences in pediatric encounters." Pediatrics111, 6-14.

Gouadec, Daniel(2010). "Quality in translation." Yves Gambier and Luc van Doorslaer (eds.) Handbook of Translation Studies: Volume 1, 270-275.

Grunwald, David and Goldfarb, Norman M.(2006). "Back Translation for Quality Control of Informed Consent Forms." Journal of Clinical Research Best Practices 2 (2). On line at: http://http://www.gtstranslation.com/medicaltranslationpaper.pdf(consulted 17.12.2013)

Heine, Paula (2003). "Best Methods for Increasing Medical Translators for Limited English Proficient Patients: The Carrot or the Stick?" Journal of Law and Health18 (1), 71.

International Medical InterpretersAssociation(2009). IMIA Guide on Medical Translation. On line at:www.imiaweb.org/uploads/pages/438.pdf (consulted 20.04.2013)

Krystallidou, Demi K.(2012). "On mediating agents' moves and how they might affect patient-centredness in mediated medical consultations." Vincent Montalt-Resurrecció and Mark Shuttleworth (eds.) (2012). Translation and knowledge mediation in medical and health settings. Linguistica Antverpiensia New Series - Themes in Translation Studies11/2012, 75-94.

Márquez Arroyo, Cristina(2007). "Interview with Henry Fischbach”. Panace 25 (8), 69-76.

Mobaraki, Mohsen and Aminzadeh, Sirwan(2012). "A Study on Different Translation Evaluation Strategies to Introduce an Eclectic Method". International Journal of English Linguistics (12/01/2012), 63-70.

Montalt-Resurrecció, Vicent and Shuttleworth, Mark(eds.) (2012). Translation and knowledge mediation in medical and health settings. Linguistica Antverpiensia New Series -Themes in Translation Studies11/2012.

Nisbeth Jensen, Matilde andKorning Zethsen, Karen(2012). "Translation of patient information leaflets: Trained translators and pharmacists-cum-translators -a comparison." Vincent Montalt-Resurrecció and Mark Shuttleworth (eds.) (2012). Translation and knowledge mediation in medical and health settings. Linguistica Antverpiensia New Series -Themes in Translation Studies 11/2012, 31-50.

O'Neil, Marla. (1998). "Who Makes a Better Translator: The Medically Knowledgeable Linguist or the Linguistically Knowledgeable Medical Professional? A Physician's Perspective." Henry Fischbach (ed.) (1998). Translation and Medicine. Amsterdam; Philadelphia: Benjamins. 